\title{
Sibling power: influence of older siblings' persuasive appeals on younger siblings' judgments about risk taking behaviours
}

\author{
Barbara A Morrongiello, Michelle DM Bradley
}

\begin{abstract}
Objectives-Although many injuries happen when school age children are away from home and in the company of other children, we know surprisingly little about interpersonal influences on children's risk taking decisions. The aim of the present study was to examine the influence of older siblings' persuasive appeals on young children's decisions about engaging in behaviours that could threaten their physical safety.
\end{abstract}

Methods-Forty same sex sibling pairs participated. Children were shown drawings of play scenes (bicycling, river crossing, and sledding), with each depicting lower and higher risk paths of travel. Children of 8 years made initial decisions as to which paths they would take. Subsequently, their older sibling acted as a confederate and tried to persuade them to change their decisions.

Results-After the appeals of older siblings, younger children significantly shifted their decisions: choices of less risky paths replaced the initial selection of more risky paths, and vice versa. A positive sibling relationship was predictive of younger siblings' decision changes. Boys and girls were equally effective in persuasion but they did so using different types of arguments, with boys communicating primarily appeals to fun and girls emphasizing appeals to safety.

Conclusions-These findings highlight the effect that older siblings can have on risk taking decisions of younger siblings. Accordingly, they document the importance of considering the interpersonal context of risk taking when designing interventions to reduce injuries among elementary schoolchildren.

(Injury Prevention 1997; 3: 23-28)

Keywords: risk taking; persuasion; siblings.

Psychology Department, University of Guelph, Guelph, Ontario N1G 2WI, Canada BA Morrongiello MDM Bradley

Correspondence and reprint requests to: Dr Morrongiello. for themselves whether or not to engage in behaviours that could threaten their physical safety. Not surprisingly, therefore, many injuries among school age children happen when they are with other children. Indeed, some have suggested that school age children are at greatest risk of injury when in the company of other children. ${ }^{34}$ Surprisingly, very little is known about interpersonal influences on school age children's decisions to engage in or avoid injury risk behaviours. Toward this aim, the present study examines the extent to which the persuasive appeals of older siblings influence younger children's decisions about engaging in behaviours that could lead to injury.

The powerful effect of peer and family influences on adolescent's adoption of health risk behaviours (for example, smoking, drug use) is well documented. ${ }^{5}{ }^{6}$ For younger children, however, much less is known about how significant others influence such decisions. A number of studies on the effects of persuasive appeals in non-risk contexts indicate that interpersonal influences on decision making operate at very young ages, ${ }^{7-9}$ with children's persuasive strategies well developed by 8 years of age. ${ }^{10}$ Furthermore, the effectiveness of persuasive appeals by elementary schoolchildren depends, in part, on the relationship shared by the persuader and the recipient child. ${ }^{11}$ For example, appeals to friends are more likely to result in compliance than are appeals to acquaintances. ${ }^{8}$

In the present study we examined how effectively children persuade other children to alter their decisions regarding behaviours that could threaten their physical safety. Because siblings have a significant influence on one another's lives, with strong emotional attachment, ${ }^{12}$ we elected to have an older sibling act as the confederate and attempt to persuade their younger sibling to do something other than what the younger child had originally planned (that is to take or avoid an injury risk situation). Furthermore, because widely spaced siblings do not have as much conflict in their relationships as closely spaced siblings, ${ }^{13}$ we selected sibling pairs that were three to four years apart in age. We limited our sample to same sex siblings because they tend to share more companionship than mixed sex pairs ${ }^{1415}$ and to identify more with one another. ${ }^{12}$ All of these are attributes we deemed important to promote consideration by the target child of the persuasive appeals offered by the confederate. ${ }^{16}$ is necessary and many injuries result from lack of knowledge, negligence, and poor judgment on the part of their caregivers. School age children, however, are increasingly left to decide on their own activities, and to judge 


\section{Methods}

SUBJECTS

The sample was recruited through the schools and comprised 40 families, including 19 having female children and 21 having male children. One of the 19 families with girls comprised twins, resulting in 20 younger girls providing data and 19 older siblings. Of the 21 families with boys, one younger boy did not complete testing.

Nearly all of the older siblings were first born and there were no siblings between the participating children. The mean (SD) ages of the younger and older children were 8.1 $(0.6)$ years and $11.3(0.6)$ years, respectively. None of the children had ever experienced an injury requiring medical care while performing the play activities included in this study. There were no age or sex differences in the number of injuries reported by parents as occurring during the past year and requiring treatment by parent (mean 8.5 ) or by a physician (mean $0.5)$.

\section{DESIGN}

To examine the persuasion process we selected three activities that were common among children in our community: bicycling, outdoor play near water, and sledding. We then developed three scenes, one involving each of these activities, that allowed for natural variations in risk to be incorporated into each scene. In each there were low and high risk paths that could be taken. Younger children initially indicated which path they would take in each scene. Following the persuasive appeals of their older sibling, they made a final decision as to which path they would follow. To examine the possibility that other factors might influence path decisions, children also assigned ratings to indicate how much risk to their safety each path posed (that is a danger rating), noted which risk factors were present in each scene,

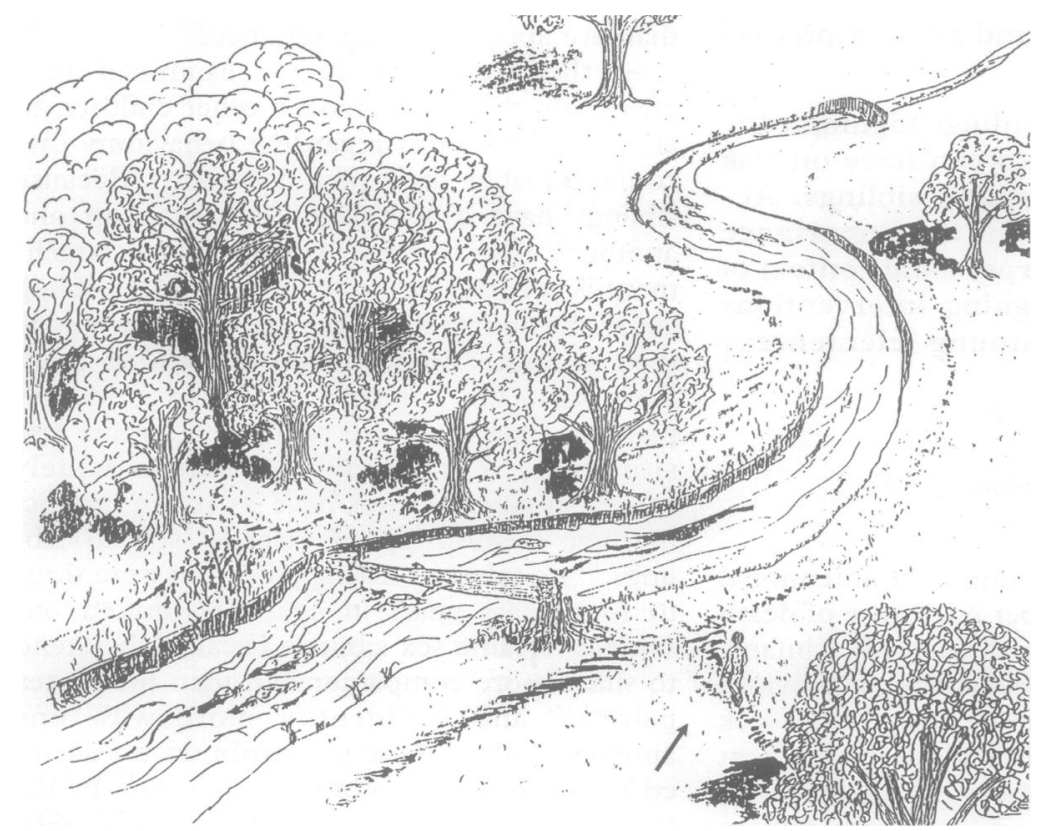

Drawing showing child (arrowed) walking to tree house on other side of stream. and indicated which paths they believed their mother and father would like them to take. Older and younger children also independently completed questionnaires to assess dimension $\overline{\bar{s}}$. of their relationship with one another and determine if the nature of the relationship affected the success of the sibling's efforts at persuasion.

\section{STIMULUS MATERIALS}

The stimuli comprised three black and white line drawings, which were extremely rich irw detail. ${ }^{17}$ An example is provided in the figures (copies are available from the first author). The stimuli were copied onto $8 \times 10$ inch card stocks paper, and then laminated. There was on drawing for each of the three types of activities bicycling through the neighbourhood to $\overrightarrow{2}$ friend's house, walking to a tree house on thes other side of a stream, and sledding down steep hill. Each drawing depicted low and high risk paths that could be taken to get from wheres the child was in the drawing to where the chils wished to go, as specified by a story told to the children. For example, for the stream crossinge depicted in the figure, the bridge was designated the low risk path and the log the high rislo path. The other two drawings incorporate of three levels of risk (low, moderate, and high risk paths).

We also made a 'danger meter' for the children to indicate how much risk of injury there was for each path in each scenario. This fabric covered cardboard $(20 \times 20 \mathrm{~cm})$ took the form of a thermometer with a zipper-type mechanism that allowed the child to slide ribbon, similar to the mercury in a thermo meter, to indicate his or her rating of risk. seven point scale was used, with 1 correspond ing to 'safe' (that is the ball end of the meter and 7 corresponding to 'very dangerous' (thas is the top end), with equidistant points along the meter.

\section{MEASURES}

The Children's Injury History Questionnaire provided demographic information on injury experiences, with an 'injury' defined as anter event (for example a cut, bump, or bruise) that made the child cry or complain of pain. This five minute questionnaire provided an index of how many and what type of injuries the children had sustained during the past year and whether medical or home treatment was? required, as reported by the primary caregivero (see Subjects section for data summary).

To assess the sibling relationship, youngen and older siblings independently completed the Sibling Inventory of Behaviour-Revised (SIB-R, ${ }^{18}$ ) and the Sibling Relationship Ques tionnaire-Revised (SRQ-R, ${ }^{14}$ ). The SIB-R is की 34 item questionnaire that provides a standar dized measure of how often children direct믄 specific positive and negative behaviours towards their sibling; interitem reliabilities range from 0.64 to $0.89 .{ }^{18}$ Young children listened to a statement (for example 'Gets angry with you'), and then rated how often their sibling 
behaved that way towards them using a five point scale that ranged from 'never' (score of 1) to 'always' (score of 5), with 'sometimes' as the midpoint. Older children read the items themselves.

The SRQ-R is a 38 item questionnaire that taps 12 dimensions of the sibling relationship and can be collapsed to yield an index both of positive and negative behaviours between the siblings. The ratings obtained by the SRQ-R relate to sibling interactions in a wide range of social contexts, ${ }^{15}$ providing support for the ecological validity of the measure. Younger children listened to each question (for example, 'How much does [name of sibling] tell you what to do?') and then chose a response from five alternatives ranging from 'hardly at all' (score of 1) to 'very very much' (score of 5), with 'somewhat' as the midpoint. Older children read the items themselves.

\section{PROCEDURES}

All test sessions were audiotaped in the homes of the participants during a two hour visit. The parent completed an Injury History Questionnaire for both the younger and older child, and answered a few questions about demographic information.

Initially, each child worked independently with different experimenters. The older child selected the paths he or she would take for each scenario, then provided a danger rating of all paths, discussed perceived risk factors for each path, and indicated which path they thought their mother and father would like them to take.

The younger sibling was presented with the scenarios in the same order as that of the older sibling, with the order randomized across sibling pairs. While the younger sibling made their path decision for the first scenario, the older sibling was instructed on their role as a confederate to persuade the younger child to alter his or her decision using whatever appeals they felt would be effective.

After the younger child made their path choice, the older sibling assumed their role as a confederate and entered the room under the pretence of waiting for the experimenter to help them with a task and he or she was secretly told which path the younger sibling had selected. The experimenter explained what the younger child was doing and the older sibling then offered to the child his or her opinion on what path should be taken, and why. The older child attempted to persuade the younger child to alter their decision without indicating knowledge of the child's original decision or familiarity with the stimulus materials. The experimenter did not participate in this discussion between the children. Following the persuasion efforts, the older sibling left the room with the experimenter and another experimenter entered. The younger child was then asked by the new experimenter to make their final decision as to which path they would take. The new experimenter continued on to obtain an initial path decision for the second scenario.
The process of alternating experimenters, with persuasion efforts by the older sibling interspersed, continued until final path decisions for all scenarios had been obtained. This allowed children to alter their path decisions without knowledge of this by their older sibling or the experimenters. This was important to avoid eliciting any concerns by younger siblings about what others would think if they altered their decisions, since the latter might result in resistance to change their decisions. Following completion of the path selection phase, younger children were asked to assign a danger rating for each path, note risk factors on each path, and to indicate which path their mother and father would want them to take.

The SRQ-R and SIB-R questionnaires were completed independently by each sibling after finishing the persuasion session. At the conclusion, all children were debriefed and given small gifts. During debriefing, none of the children expressed any awareness of a pattern regarding which paths the older sibling was encouraging them to take. In fact, most younger children expressed surprise when the role of their older sibling as a confederate was explained.

\section{DATA REDUCTION}

Initially, audiotapes were transcribed verbatim. To examine if number of risk factors identified varied systematically with age and/or sex, we first made a list of which factors were reliably mentioned by at least $20 \%$ of the children and less frequently mentioned factors were excluded from analyses. Next, we computed a proportion score for each individual for each path: the number of risk factors identified for a path was divided by the total number mentioned across all children for that path. These proportion scores were then statistically analyzed.

Each child was assigned a score depending on which path was selected for each scenario, and scores were then analyzed to assess for age and/or sex differences.

To examine the persuasion process, two raters independently identified discrete persuasive appeals, achieving $94 \%$ agreement $(\kappa$ statistic), with remaining disagreements resolved by discussion. Subsequently, we categorized the type of persuasive appeal made by older siblings into one of four categories derived from the data: (1) safety based arguments (for example 'There are fewer cars to worry about that way'), (2) convenience based arguments (for example, 'That way is faster'), (3) fun based arguments (for example 'That way is more exciting'), and (4) 'other'. A second scorer categorized $25 \%$ of the persuasive appeals, yielding a reliability estimate of $97 \%$ ( $\kappa$ statistic). The categorizations of the primary coder were analyzed.

For the SIB-R and the SRQ-R we computed a ratio score to reflect the balance of positive to negative attributes in the sibling relationship, with a score above 1.0 indicating a predominantly positive relationship and a score below 
1.0 indicated more negative than positive attributes. Because a Pearson correlation between these two ratio scores revealed a high degree of redundancy $(r=0.89, \mathrm{p}<0.0001)$, we averaged the ratio scores for each subject, thereby obtaining one score that reflected the balance of positive to negative characteristics in the sibling relationship. These data were then related to number of path decision changes.

\section{Results \\ CHILDREN'S PATH DECISIONS \\ Before persuasive appeals}

Table 1 shows the initial path decisions of the children as a function of scenario, sex, and age group. As can be seen, for the bicycling scenario, at both ages, females were more inclined than males to select the moderate risk path, whereas males more often than females chose the high risk path $(p<0.05)$. For the sledding and river crossing scenarios, the same trend for males to select the higher risk path more often than females was evident for younger children for the sledding and river crossing scenario, and for the older children for the river crossing scenario. Similarly, when collapsed over the three scenarios and two ages, males selected the higher risk path more often than females $\left(\chi^{2}=3.80, p<0.05\right)$. Thus, consistent with the higher incidence of injuries among boys in the literature, boys were more likely than girls to select routes that posed a greater threat of injury.

All children were asked to predict which paths in each scenario their mothers and fathers would prefer them to take. Many children $(68 \%)$ selected paths they felt their parents would allow them to travel on, but $80 \%$ of those who selected 'high risk' paths indicated an awareness that their parents would not want them to do so. When the children explained this discrepancy, they typically $(82 \%)$ said that the parent did not know how good they were at the activity depicted; in other words, the children often provided a competency based rationale for their selection of the 'high risk' path. Interestingly, when there were discrepancies between what the children believed their mother and father would allow them to do $(37 \%)$, fathers were nearly always (91\%) perceived as willing to allow the child to take a higher risk path then would the mother.

After persuasive appeals

Table 2 shows the per cent of decision changes from low to higher risk, and vice versa, by

Table 1 Percentage of boys and girls in each age group (young, old) that initially chose each path (low, medium, high risk) in each scenario

\begin{tabular}{|c|c|c|c|c|c|c|c|c|}
\hline \multirow{2}{*}{$\begin{array}{l}\text { Group/ } \\
\text { sex }\end{array}$} & \multicolumn{3}{|c|}{ Bicycling } & \multicolumn{3}{|c|}{ Sledding } & \multicolumn{2}{|c|}{ River crossing } \\
\hline & Low & Medium & High & $\overline{\text { Low }}$ & Medium & High & Low & High \\
\hline $\begin{array}{l}\text { Young } \\
\text { Girls }(n=20) \\
\text { Boys }(n=20)\end{array}$ & $\begin{array}{l}15 \\
25\end{array}$ & $\begin{array}{l}75 \\
30\end{array}$ & $\begin{array}{l}10 \\
45\end{array}$ & $\begin{array}{l}55 \\
75\end{array}$ & $\begin{array}{l}30 \\
0\end{array}$ & $\begin{array}{l}15 \\
25\end{array}$ & $\begin{array}{l}75 \\
60\end{array}$ & $\begin{array}{l}25 \\
40\end{array}$ \\
\hline $\begin{array}{l}\text { Old } \\
\text { Girls }(n=19) \\
\text { Boys }(n=21)\end{array}$ & $\begin{array}{l}11 \\
19\end{array}$ & $\begin{array}{l}84 \\
52\end{array}$ & $\begin{array}{l}5 \\
29\end{array}$ & $\begin{array}{l}32 \\
52\end{array}$ & $\begin{array}{l}32 \\
19\end{array}$ & $\begin{array}{l}36 \\
29\end{array}$ & $\begin{array}{l}74 \\
67\end{array}$ & $\begin{array}{l}26 \\
33\end{array}$ \\
\hline
\end{tabular}

younger siblings. Of the 40 younger children, $22(55 \%)$ changed their decisions for at least one scenario. These 22 children made a total of 35 decision changes across the three scenarios. (48\% change rate), and there were no sig-0 nificant sex differences in the number of decision changes.

As can be seen in table 2, collapsing ovet? scenarios, following the persuasive appeals of their older siblings, younger children signifi 흠 cantly altered their decisions from higher to lower risk paths $(t(31)=4.31, \mathrm{p}<0.001)$, and from higher to lower risk ones $(t(31)=7.19 \%$ $\mathrm{p}<0.005)$. Comparing the rates of decision 5 changes from lower to higher risk, and vice versa, within each scenario, revealed compar able significant results $(\mathrm{p}<0.05)$.

\section{Sibling relationship and decision changes}

To examine if the nature of the sibling relationship was related to the likelihood of the younger children altering their path deci sions, a one tailed bivariate regression analysis was conducted using sibling relationship scores to predict number of decision changes. Resultई revealed that younger children's positive perceptions of their relationship with their oldeb sibling predicted decision changes regardless of the direction of shift $(r=0.34, \mathrm{p}<0.05)$. Thus children were more susceptible to influence् when the relationship they shared with the persuader was predominantly positive (that is acceptance, kindness, prosocial, nurturance affection, companionship, intimacy, admira tion, similarity) as opposed to negative (that is. dominance, quarrelling, competition, aggres sion, hostility, avoidance).

\section{Types and number of persuasive appeals}

To examine whether there were differences in the type of persuasive appeals expressed by boys and girls for the different scenarios multivariate analysis of variance was conducte $\$$ with sex (2), scenario (3), and type on persuasive appeal (4) as factors. As is seen in table 3, the type of argument expressed varied depending on sex $(F(3,114)=3.32, p<0.05)$ ․․․ A Newman-Keuls analysis revealed that boys made significantly fewer appeals to safety and significantly more appeals to fun-related out: comes in comparison to girls $(p<0.05) \omega$ Although one might expect that the type of persuasive argument would vary with the context, the incidence and type of appeal did not vary across scenario $(p>0.05)$.

Finally, a Pearson correlation between the

Table 2 Percentage of decision changes (number of each is indicated within parentheses) for each scenario by sex Decisions could change from lower to higher risk paths (designated by a '+'), or from higher to lower risk pathe (designated by $a$ '-')

\begin{tabular}{|c|c|c|c|c|c|}
\hline \multirow[b]{2}{*}{ Sex } & Bicycling & Sledding & \multicolumn{2}{|c|}{ River crossing } & Total \\
\hline & $\mp$ & 7 & + & - & - \\
\hline Girls & 1000 & $80 \quad 20$ & 71 & 29 & 8416 \\
\hline Boys & ${ }_{(6)}^{50} 50$ & ${ }_{(8)}^{38} 62$ & $\begin{array}{r}62 \\
(8)\end{array}$ & 38 & $\begin{array}{c}68 \text { (22) } \\
32\end{array}$ \\
\hline
\end{tabular}


Table 3 Proportion of persuasive appeals of each type that older boys and girls made to their same sex younger siblings

\begin{tabular}{lllll}
\hline \multirow{5}{*}{ Sex } & \multicolumn{4}{l}{ Type of persuasive appeal } \\
\cline { 2 - 5 } & Safety & Convenience & Fun & Other \\
\hline Girls & 0.53 & 0.25 & 0.22 & 0.00 \\
Boys & 0.20 & 0.24 & 0.48 & 0.08 \\
\hline
\end{tabular}

number of decision changes $(0-3)$ and total number of arguments revealed that the more arguments that were made the more likely children were to change their decisions $(r=0.59, \mathrm{p}<0.001)$. Thus, it was not just what was said but the number of arguments made that influenced the effectiveness of persuasive appeals by children.

\section{RISK PERCEPTION}

Identifying dangers

Children were asked to identify things that could be dangerous for them on each of the paths in the three situations. An analysis of variance indicated no age or sex differences in the number of dangers mentioned $(p>0.05)$. Children were careful observers and mentioned not only the more obvious hazards that posed the greatest risks, but a number of other less significant hazards as well, averaging two to four dangers per path.

\section{Danger ratings of paths}

Children also assigned danger rating scores (range: $0-7$ ) to each path. An analysis of variance with age (2), sex (2), scenario (3), and path (2) as factors revealed that children's ratings of danger for the different paths varied for the low and high risk routes $(F(1$, 474) $=628.14, p<0.001)$. Paired comparison tests confirmed, as shown in table 4 , that children assigned significantly different danger ratings for paths within each scenario and these ratings followed the expected ordering of paths with respect to degree of injury risk $(p<0.01)$.

It is noteworthy, however, that the number of dangers children mentioned did not correlate significantly $(p>0.05)$ with their danger ratings for each path. It was not the number of hazards per se that influenced the danger ratings, but the nature of what the hazards were. Even for the highest risk paths children often mentioned only one to three hazards, although their danger ratings indicate that they weighted these hazards as posing more of an injury threat than the hazards they identified for the lower risk paths.

\section{Danger ratings and path decisions}

Pearson correlations were conducted to determine if the danger ratings related to path decisions. For older children, there was only one relationship that was significant, suggesting that older children were influenced by factors other than risk ratings in making their path decisions. By contrast, younger children's path decisions related systematically to their risk ratings. For each of the scenarios, the
Table 4 Average danger ratings (possible range: 0-7) children assigned for each path in each scenario

\begin{tabular}{lllllll}
\hline Bicycling & & \multicolumn{2}{l}{ Sledding } & & \multicolumn{2}{l}{ River crossing } \\
\cline { 4 - 5 } \cline { 5 - 6 } funkyard & Street & & Rocky & Smooth & Log & Bridge \\
\hline 5.0 & 2.0 & 5.6 & 2.0 & 5.1 & 1.9 \\
\hline
\end{tabular}

higher the danger rating assigned a path the less likely younger children were to chose that path $(r=-0.37,-0.58$, and -0.32 , respectively, for the high risk paths, $\mathrm{p}<0.001$ ).

\section{Discussion}

In this study, boys generally selected a high risk path more often than girls. Increased risk taking by males is consistent with injury statistics: boys have two to four times more injuries than girls. Although the parents of boys in our sample did not report higher injury rates, this probably reflects inaccurate measurement of these rates by having parents provide this information on behalf of their 8 and 11 year old children. Children in this age range sometimes report injuries of which parents are not aware. ${ }^{19}{ }^{20}$ For elementary schoolchildren, therefore, parents may not be as well informed as the children are when it comes to reporting on injuries, particularly minor ones.

Elementary school age children were susceptible to the influence of older children's persuasive appeals for risk taking. Not surprisingly, a positive relationship between the two resulted in children being more vulnerable to persuasion. ${ }^{1121}$ Extending these results to peer relationships, it is likely that children will more readily be influenced to follow the lead of best friends rather than acquaintances or friends with whom they do not share a predominantly positive relationship. ${ }^{8}$ The finding that boys and girls expressed different types of arguments (predominantly safety based by girls and fun appeals by boys), though they shared the same goals, suggests that interventions aimed at inoculating or training children to resist peer pressures for risk taking, ${ }^{22}$ may have to differ in content for boys and girls in order to be effective.

The finding that the number of persuasive appeals influenced children's making a decision change indicates that what is said, as well as how many arguments are made, both influence elementary schoolchildren being persuaded to alter a decision about risk taking. In this study we focus exclusively on what the older sibling said to the target child. However, these dyads typically became involved in discussion, exchanging information about the pros and cons of each path. Possibly, in this context, which allowed participants to engage in dialogue about the risk taking situation, the number of arguments has a cumulatively greater influence. Related to this, although we studied persuasion as though it were a unidirectional process (older sibling influencing younger child), we do not assume this to be the 
case. In fact, we think it more parsimonious to think of persuasion as a bidirectional process because dyads who share a meaningful relationship are likely to reciprocally exchange information in attempting to bring their own opinion to bear and to influence decision making. A study in progress in which we are examining the process of persuasion within best friend dyads, and are attempting to code the exchanges so as to capture more about the process that leads to successful persuasion outcomes, should provide further insights into this issue.

Not surprisingly, younger children's risk taking decisions related to their danger ratings for the different paths. The more a path was rated as dangerous the less children selected it for travel. At older ages, however, there was no statistical relationship between risk taking decisions and danger ratings, suggesting that other factors influenced older children's risk taking decisions. Obviously, research on what factors influence children's risk taking decisions at different ages is needed. For adults, decisions about risk taking are influenced by several factors such as: perceived personal vulnerability or susceptibility to that danger, beliefs about the incidence or prevalence of injury, and a weighting of the costs (for example, injury) to potential gains (for example, faster) of engaging in the risk taking activity. ${ }^{23}$ Perhaps, with increasing age, children also come to consider these factors, resulting in risk taking becoming increasingly multidetermined with increasing age.

Several of our findings suggest other interesting questions to pursue in future research. For example, children consistently reported a belief that their father would tolerate their engaging in more risk taking than their mother. Relatively little is known, however, about the role, if any, parents have in promoting risk taking in their children, or if mothers and fathers differ in their tolerance for risk taking. A second question that merits further study emerges from our finding that children who selected paths higher in risk than they expected their parents to tolerate often explained their decision by alluding to a competency level that they felt was greater than that for which their parent gave them credit. This result suggests that children are aware of injury risks, which is consistent with their differential risk ratings for the paths (see table 4), but that they provide for themselves rational and valid reasons to support their risk taking decisions. To the extent then that children have unrealistic expectations of their abilities, they may be especially likely to place themselves at risk for making poor decisions that lead to increased injury risk.
Extending this reasoning, if males are more likely than females to overestimate their abilities, this also could explain greater risk taking by males than females leading to their greate $\overline{\text {. }}$. injury rates.

This research was supported by grants to the first author from the Hospital for Sick Children Foundation and the Socia Sciences and Humanities Research Council. The authors thank the families who participated for their interest and enthusiasn? the families who participated for their interest and enthusiasme Dr Susan Lollis for comments on an earlier version of the
manuscript, and Colleen Hoey and Tess Dawber for assistance manuscript, and Colleen Hoey and Tess
with data reduction, coding, and analysis.

1 Baker SP, O'Neill B, Karpf RS. The injury fact book Lexington, MA: Lexington Books, 1983.

2 Canadian Institute of Child Health. The health of Canada; children. 2nd Ed. Ottawa: CICH, 1994.

3 Wilson M, Baker S, Teret S, Shock S, Garbarino J. Saving children: a guide to injury prevention. New York: Oxford

4 Sandels S. An overall view of children in traffic. In: Jackson $\mathrm{R}$, ed. Children, the environment, and accidents. Kent, England: Pitman Medical, 1977.

5 Brook JS, Whiteman M, Gordon AS, Brook DW. The role of older brothers in younger brother's drug use viewed in the context of parent and peer influences. $\mathcal{f}$ Genet Psychol. 1990; 151: $59-75$.

6 Quine S, Stephenson J. Predicting smoking and drinkin intentions and behavior of pre-adolescents: the influence्f of parents, siblings and peers. Family Systems Medicins 1990; 8: 191-200.

7 Haslett B. Preschooler's communicative strategies in gainin $\overrightarrow{0}$ compliance from peers: a developmental study. Quarter fournal of Speech 1983; 69: 84-99.

8 Jones DC. Persuasive appeals and responses to appeals among friends and acquaintances. Child Dev 1985; 56 $757-63$

9 Williams D, Shaller A. Peer persuasion: a study of children' dominance strategies. Early Child Development and Carg 1993; 88: $31-41$.

10 Levin E, Rubin K. Getting others to do what you want then to do: the development of children's requestive strategies In: Nelson K, ed. Children's language. Vol 4 . New Jersey Erlbaum, 1983.

11 Bigelow BJ, Tesson G, Lewko JH. The social rules that children use: close friends, other friends, and 'other kids? compared to parents, teachers, and siblings. Internation fournal of Behavior and Development 1992; 15: 315-35.

fournal of Behavior and Development 1992; 15: $315-35$.
12 Dunn J. Sibling relationships in early childhood. Child Deף 1983. 54: 787 relations

13 Minnett AM, Vandell DL, Santrock JW. The effects of sibling status on sibling interaction: influence of birt? order, age spacing, sex of child, and sex of sibling. Chil\$ Dev 1983; 54: 1064-72.

14 Furman W, Buhrmester D. Children's perceptions of the personal relationships in their social networks. Develop $\overline{0}$ mental Psychology 1985; 21: 1016-24.

15 Furman W, Buhrmester D. Children's perceptions of the qualities of sibling relationships. Child Dev 1985; 56. qualities

16 Miller GR, Boster F. Persuasion in personal relationshipsô In: Duck SW, ed. A handbook of personal relationships In: Duck SW, ed. $A$ handbook of persom

17 Christensen $S$. The effect of peers on children's judgements abou engaging in behaviours that threaten their safety. (Master's thesis.) Guelph, Ontario: University of Guelph, 1993.

18 Schaeffer E, Edgerton M. The sibling inventory of behavior (Unpublished manuscript.) Chapel Hill NC: University

19 Peterson L, Harbeck C, Moreno A. Measures of children's injuries: self reported versus maternal-reported events with temporally proximal versus delayed reporting.
Pediatr Psychol 1993; 18: 133-47.

20 Morrongiello BA. Children's perspectives on injury an close call experiences: sex difference in injury-outcome processes. $¥$ Pediatr Psychol (in press). processes. F Pediatr Psychol (in press)

21 Clark R, Delia J. The development of functional persuasiv $\mathbb{E}^{\circ}$ skills in childhood and early adolescence. Child Dev 1976 47: $1008-14$.

22 Duryea EJ, Ransom MV, English G. Psychological immunization: theory, research, and current health behaviou? applications. Health Educ $Q$ 1990; 17: 169-78.

23 Janz N, Becker $M$. The health beliefs model: a decade later Health Educ $Q$ 1984; 11: 1-47. 